\title{
Loss of intermediate flow states only evident when considering sub-daily flow metrics in a major tributary in the Limpopo basin
}

\author{
Pfananani Ramulifho ${ }^{1}$, Nick Rivers-Moore ${ }^{2}$, and Stefan Foord ${ }^{1}$ \\ ${ }^{1}$ University of Venda \\ ${ }^{2}$ University of KwaZulu-Natal School of Life Sciences
}

July 20, 2021

\begin{abstract}
As the impacts of the anthropocene intensifies in rivers, there is an increasing need to understand how these changes affect both daily and sub-daily stream flow variability, timing and flow quantities, as these are some of the most influential drivers of spatial and temporal dynamics of stream biota. In this paper, long-term changes in flow patterns of a strategic water source area in an arid region of southern Africa were quantified, focusing on the relation between daily and sub-daily and its potential impact on fish biota of the catchment. Long-term temporal trends in stream flow were modelled using Generalized Least Squares (GLS), while sub-daily and daily mean flow of the same stations were compared using a suite of metrics. Periods of similar stream flow patterns were identified using K-means cluster analysis. A spreadsheet rule-based model was developed linking fish communities to streamflow patterns providing a predictive framework for fish assemblage responses to stream flow classes. Long term reduction in flow has a strong seasonal component, with significant decreases during the wet season, not linked to long-term rainfall patterns. Flow variability has increased over time, while $78 \%$ of sub-daily flow metrics were related to daily flow metrics. Oscillating flow conditions and the loss of intermediate flow states may permanently exclude certain fish flow guilds. However, temporal partitioning is only evident when sub-daily metrics are considered, highlighting their importance for assessing ecological resilience.
\end{abstract}

Hosted file

Revised Paper_sub-daily flow metrics.docx available at https://authorea.com/users/426512/ articles/531003-loss-of-intermediate-flow-states-only-evident-when-considering-subdaily-flow-metrics-in-a-major-tributary-in-the-limpopo-basin 